\title{
POSSIBLE ORDERS OF TWO GENERATORS OF THE ALTERNATING AND OF THE SYMMETRIC GROUP*
}

\author{
BY \\ G. A. MILLER
}

It is well known that every alternating and every symmetric group can be generated by two of its substitutions, and that two such generating substitutions can usually be selected in a large number of different ways. Since two operators of order 2 must always generate a dihedral group it is evident that no alternating group can be generated by two of its substitutions of order 2 , and that the only symmetric group which can be thus generated is the symmetric group of order 6 . On the other hand, it is known that with very few exceptions, relating to groups whose degrees do not exceed 8, every alternating group and every symmetric group can be generated by two of its substitutions of orders 2 and 3 respectively. $\dagger$ In the present article we shall prove that whenever an alternating group involves a substitution of order $l>3$ then it contains two substitutions of orders 2 and $l$ respectively which generate the entire group. We shall also determine the degrees of all the symmetric groups to which a similar theorem does not apply.

Before proving this general theorem, it may be desirable to consider the more elementary question of generating an alternating or a symmetric group by two of its substitutions which are separately composed of a single cycle. When neither of the two numbers $l_{1}, l_{2}$ exceeds $n$ but their sum exceeds $n$ it is obvious that two substitutions $s_{1}, s_{2}$ which are separately composed of a single cycle, and whose orders are $l_{1}, l_{2}$ respectively, can be so selected that they generate a transitive group of degree $n$, and that half the substitutions of this transitive group are negative whenever at least one of the two numbers $l_{1}, l_{2}$ is even. If $s_{1}$ and $s_{2}$ do not have all their letters in common we may suppose that the common letters are arranged in the same order in both of these substitutions and hence their commutator is either of the form $a b c$ or of the form $a b \cdot c d$. If both of them are of degree $n$ we may suppose that all their letters are arranged in the same order with the exception that two adjacent letters are interchanged. Hence their commutator is of the form $a b c$ in this case. The group generated by $s_{1}, s_{2}$ is obviously multiply transitive

* Presented to the Society, December 31, 1926; received by the editors, December 20, 1926.

† G. A. Miller, Bulletin of the American Mathematical Society, vol. 7 (1901), p. 424. 
whenever $n>3$ and hence it must be alternating or symmetric, at least when $n>8$, since the class of a primitive group which is neither alternating nor symmetric must exceed 4 whenever its degree exceeds 8 . When $n$ does not exceed 8 it is easy to verify directly that two such substitutions can be so selected as to generate the alternating groups when both of the numbers $l_{1}, l_{2}$ are odd, and the symmetric group when at least one of these numbers is even. These results may be stated in the form of a theorem as follows: If $l_{1}, l_{2}$ represent a pair of numbers, each being greater than unity, such that neither exceeds $n$ but their sum exceeds $n$, then it is always possible to find two cycles of orders $l_{1}$ and $l_{2}$ respectively such that they generate the alternating group of degree $n$ whenever both $l_{1}$ and $l_{2}$ are odd. When at least one of these two numbers is even they generate the symmetric group of the same degree.

From this theorem it results directly that if $l_{1}, l_{2}$ represents any pair of positive integers such that each exceeds unity then it is always possible to find two cycles $s_{1}, s_{2}$ of orders $l_{1}, l_{2}$ respectively such that the group generated by $s_{1}, s_{2}$ is either alternating or symmetric and has an arbitrary one of $l_{1}, l_{1} \leqq l_{2}$, different degrees. For instance, pairs of cycles of order 9 can be selected such that each pair generates an arbitrary one of nine different alternating groups, viz., the alternating groups whose degrees vary from 9 to 17 inclusive, while an arbitrary one of the nine different symmetric groups of the degrees 10 to 18 can be generated by a cycle of order 9 and a cycle of order 10 . This constitutes a complete solution of the elementary problem of generating alternating or symmetric groups by means of twocycles on their letters.

For the proof of the general theorem noted in the first paragraph it will often be convenient to use the following obvious theorem: If a transitive group of degree $n$ contains a cycle of prime order $p$, where $p$ satisfies the condition $n / 2<p \leqq n-3$, it must be either alternating or symmetric. It is known that whenever $n>7$ it is always possible to find such a prime number.*

It will also frequently be desirable to use the following theorem:

If a transitive group is generated by two substitutions $s_{1}, s_{2}$ and if one of these substitutions $s_{2}$ involves one and only one cycle of a given prime degree while the other does not transform all the letters of this cycle into letters which do not occur in it, then the transitive group generated by $s_{1}, s_{2}$ is either alternating or symmetric whenever its degree exceeds the degree of this cycle by more than 2.

Since it is well known that a primitive group of degree $n$ which does not include the alternating group of this degree can not involve a substitution

* G. A. Miller, School Science and Mathematics, vol. 21 (1921), p. 874. 
composed of a single cycle of prime degree less than $n-2$, it is only necessary to show that $s_{1}$ and $s_{2}$ generate a primitive group. To do this we may transform by $s_{1}$ a power of $s_{2}$ which is equal to this prime cycle and thus obtain a prime cycle which has not all its letters in common with the former but has at least one letter in common therewith. Hence the group $G$ generated by $s_{1}, s_{2}$ involves two such prime cycles which generate a doubly transitive group whose degree is just one larger than the degree of one of these cycles. Since a transitive group which contains a primitive subgroup of lower degree is itself primitive unless all the letters of this primitive subgroup appear in one of the sets of every one of its possible systems of imprimitivity, it results almost directly that $G$ must be primitive and hence the theorem in question has been established.

To exhibit the nature of the limitations imposed in this theorem and at the same time prove a somewhat striking theorem it may be noted here that if $s_{2}$ represents a substitution of composite order $k_{1} k_{2}$ and is composed of two cycles of orders $k_{1} k_{2}$ and $k_{1}$ respectively, and if $s_{1}$ is a transposition which involves one letter from each of these two cycles, then the group generated by $s_{1}, s_{2}$ is imprimitive and contains invariantly the direct product of $k_{1}$ symmetric groups which are separately of degree $k_{2}+1$. A proof of this theorem results from the following consideration. The commutator of $s_{1}$ and $s_{2}{ }^{k_{1}}$ is a cycle of order 3 . The symmetric group of degree 3 generated by this commutator and $s_{1}$ has two letters in common with a cycle of $s_{2}{ }^{k_{1}}$ and these two letters are adjacent in this cycle. Hence $G$ involves the symmetric group of degree $k_{2}+1$ in view of the theorem noted near the close of the preceding paragraph. This symmetric group is transformed by $s_{2}$ into $k_{1}$ symmetric groups such that no two of them have a letter in common. The order of $G$ is $k_{1}$ times that of the direct product of these symmetric groups. The simplest illustration of such a group is the transitive group of degree 6 and of order 72 . In this case $k_{1}=k_{2}=2$.

Another elementary theorem which will be very useful in the solution of our general problem may be stated as follows:

If $s_{1}, s_{2}$ generate a transitive group and if $s_{1}$ has only one letter in common with some power of $s_{2}$ and if the letter by which this common letter is replaced in $s_{1}$ does not appear in a cycle of $s_{2}$ whose order is a multiple of the number of cycles in the given power of $s_{2}$, then this transitive group includes the alternating group of its degree.

The proof of this theorem is similar to that noted in the preceding paragraph. The commutator of the given power of $s_{2}$ and $s_{1}$ is again a cycle of 
order 3 and hence $G$ involves the alternating group on a number of letters which is at least one larger than the order of a cycle in the said power of $s_{2}$. This alternating group involves letters from at most two cycles of $s_{2}$. Hence $s_{2}$ would have to transform it into alternating groups on sets of distinct letters if the theorem were not true. As this is impossible from the conditions noted in the theorem it results that the theorem is established. When the given power of $s_{2}$ is a single cycle it is clear that the condition as regards the letter by which the common letter is replaced in it may be omitted in the theorem.

In what follows $s_{2}$ will represent a substitution of order $l>3$ while $s_{1}$ will represent a substitution of order 2. The smallest possible degree of $s_{2}$ is the sum of the highest powers of the prime power factors of $l$, and when $l$ is even, $s_{2}$ must be negative both for this smallest degree and also for the next larger degree of the symmetric group in which it appears. In every larger symmetric group there is a positive as well as a negative substitution of order $l$. It will be assumed that $s_{1}$ and $s_{2}$ have been so constructed that they generate a transitive group of degree $n$, and it results directly from the theorems noted above that when $l$ is a given even number, $s_{1}, s_{2}$ can be so chosen that they generate the symmetric group when $n$ has the smallest possible value or the next larger value. In what follows we may therefore always assume that $n$ has a ląrger value. When $l$ is odd, $s_{2}$ must be positive, and when $l$ is even it will be assumed that $s_{2}$ is positive unless the contrary is stated, and that the degree of $s_{2}$ is not less than $n-p_{1}+1$, where $p_{1}$ is the smallest prime factor of $l$ when $l$ is either odd or divisible by 3 . When neither of these two conditions is satisfied then the degree of $s_{2}$ may be assumed to be at least $n-3$. Moreover, it will generally be assumed that the cycles of $s_{2}$ appear in descending order of magnitude in case there is a difference in their orders.

When $l$ is divisible by at least three distinct prime numbers it is obvious that $s_{1}$ can be so selected that it has only one letter in common with the firse cycle of $s_{2}$ and that all the other cycles may be assumed to be of lower primt power orders. Moreover, when it is desirable to add to $s_{1}$ another transposition in order to give it the suitable sign we may form this, in case the order of the first cycle is divisible by the square of a prime number, on letters of this first cycle in such a way that the commutator of this cycle and $s_{1}$ is composed of a cycle of ordet 3 and of two transpositions. The square of this commutator is therefore a cycle of order 3 and may be used just as the commutator was used in the preceding case. When the order of this first cycle is not divisible by the square of a prime number, a transposition on the letters of the first cycle of $s_{2}$ may be added arbitrarily. Hence $s_{2}$ and $s_{1}$ can 
always be so selected as to generate either the alternating group of degree $\boldsymbol{n}$ or the symmetric group of this degree, as may be desired, whenever $l$ is divisible by at least three distinct prime numbers and the group concerned contains a substitution of order $l$. When $l$ is divisible by two distinct odd prime numbers, or by one such number and 4 , the remarks which have just been made still apply, and hence we may assume in what follows that $l$ is either a power of a prime number or the double of a power of an odd prime number.

When $l=2 p_{1}$, where $p_{1}>3$ is a prime number, it results again directly from the preceding theorems that $s_{1}$ and $s_{2}$ can be so selected as to generate either the symmetric or the alternating group, as may be desired, whenever the group in question involves a substitution of order $l$. When $l=2 p_{1}^{\alpha}, \alpha>1$, and $p_{1}$ any odd prime number, it is easy to prove that $s_{1}, s_{2}$ can be so chosen that their product contains a cycle of order $p$, as defined above, and that another transposition can be added to $s_{1}$ so as to give it the proper sign without affecting this cycle of order $p$. A simple proof of this fact may be given as follows. First, select $s_{1}$ so as to connect the last letter of the first cycle of $s_{2}$ with the first letter of the second cycle, and the first letter of every other cycle with the second letter of the preceding cycle. When the degree $s_{2}$ is not $n$, we connect also the last letter of $s_{2}$ with a letter not found in $s_{2}$, and when $n$ exceeds the degree of $s_{2}$ by more than 1 we may connect the additional letter or letters with the second or the second and third letter of the first cycle of $s_{2}$. It was noted above that there could not be more than two such additional letters. When $s_{1}^{\prime}$ is selected in this way it is obvious that $s_{1}^{\prime} s_{2}$ is a single cycle of order $n$. If the $(p+1)$ th letter of this cycle, counting from the next to the last letter of the first cycle of $s_{2}$, is not in $s_{1}^{\prime}$, we add to $s_{1}^{\prime}$ the transposition composed of this letter and the next to the last letter of the first cycle in $s_{2}$. The product of $s_{2}$ and the $s_{1}$ thus obtained will then involve a cycle of order $p$ and this cycle will not be affected by adding a properly chosen transposition to $s_{1}$ to give it the desired sign.

It remains to consider the case when the $(p+1)$ th letter of the given cycle of order $n$ appears in $s_{1}^{\prime}$. If in this case the letter which precedes this $(p+1)$ th letter does not appear in $s_{1}^{\prime}$ we start our cycle with the third letter from the end of the first cycle of $s_{2}$ and proceed as before. If both the $(p+1)$ th letter and the preceding letter of the cycle of order $n$ appear in $s_{1}^{\prime}$ and $p_{1}>3$ we start our cycle with the sixth letter from the end of the first cycle of $s_{2}$ and proceed as before. If $p_{1}=3$ we start with the fourth letter from the end of this cycle. If $s_{2}$ has been so chosen that it involves as small a number of transpositions as possible, no other case can present itself and hence it remains 
to consider only the cases when $l=6$, and when $l$ is a power of a single prime number.

When $l=6$ and $s_{2}$ is positive, $n>6$. When $n=7$ or 8 , it follows directly from the general theorems noted above that $s_{1}, s_{2}$ can be suitably selected. When $n>7$ it may be assumed that the first cycle in $s_{2}$ is of order 6 and that $s_{2}$ involves no more than one transposition. It may also be assumed that its degree is not less than $n-2$, since cycles of order 3 may be added to it if necessary to increase its degree. Hence it is obvious that $s_{1}$ and $s_{2}$ may be so selected that their product involves a cycle of order $p$ and that $s_{1}$ is either positive or negative as may be desired. It remains to consider the case when $l$ is a power of a single prime number and $l>3$.

The case when $l=p_{1}, p_{1}$ being a prime number, is especially interesting since there is an infinite number of values of $n$, one and only one for each such prime number, such that the symmetric group of degree $n$ contains a substitution of order $l$ but cannot be generated by two operators of orders 2 and $l$ respectively. The fact that $2 p_{1}-1$ is such a value of $n$ is obvious since $s_{1}$ must then be of degree $2 p_{1}-2$ and hence it must be positive. This substitution and $s_{2}$ generate the alternating group of degree $n$ according to the general theorems noted above, and hence it remains to prove that for every other value of $n$ it is possible to find two substitutions of orders 2 and $l$ respectively which generate either the alternating group or the symmetric group of degree $n$ as may be desired. When $n<2 p_{1}$, this requires no further proof since it is included in a general theorem noted above. When $n \geqq 3 p_{1}$ it is obvious that $s_{1}, s_{2}$ can be so chosen that their product involves a cycle of order $p$. When $n=2 p_{1}+k, k<p_{1}$, we may first consider the case when $k=p_{1}-1$. If $p>n-p_{1}^{\prime}$, it must be at least equal to $n-p_{1}+2$. Hence we may connect the letters of the second cycle of $s_{2}$ by means of $s_{1}^{\prime}$ with the $k$ letters which do not appear in $s_{2}$ and add a suitable transposition to $s_{1}$ so as to obtain a cycle of order $p$ in the product of $s_{1}^{\prime}$ and $s_{2}$. An additional transposition on the letters of the first cycle of $s_{2}$ may be added to $s_{1}^{\prime}$ so as to give it the desired sign without affecting this cycle of order $p$, since $p$ cannot exceed $n-3$. When $p=n-p_{1}$, then we may connect one of the letters not found in $s_{2}$ with the first letter in the first cycle of $s_{1}$ and the other letters not found in $s_{2}$ with letters of the second cycle of $s_{2}$. When $p<n-p_{1}$ we can evidently proceed in a similar way. Finally, when $k<p_{1}-1$ we may again connect by $s_{1}$ the letters of the second cycle of $s_{2}$ with those not found in $s_{2}$ and thus obtain suitable forms for $s_{1}$ and $s_{2}$. Hence the following theorem. If $l>3$ represents a prime number which divides the order of the symmetric group of degree $n \neq 2 l-1$, then it is always possible to find two operators of orders $l$ and 2 respectively which generate this symmetric group and also two such operators 
which generate the alternating group of this degree. When $n=2 l-1$ it is possible to find two such generators of the alternating group of degree $n$ but it is impossible to find two such generators of the symmetric group of this degree.

When $l=p_{1}^{\alpha}$, where $p_{1}$ is any odd prime number and $\alpha>1$, the substitution $s_{2}$ may be assumed to be of degree $n-k, k<p_{1}$. If $s_{2}$ involves more than one cycle we may again suppose that $s_{1}^{\prime}$ connects the last letter of the first cycle of $s_{2}$ with the first letter of the second cycle and the first letter of every other cycle with the second letter of the preceding cycle. Moreover, $s_{1}^{\prime}$ connects letters of the last cycle in $s_{2}$ with the $k$ letters which do not appear in $s_{2}$. The product $s_{2} s_{1}$ is a cycle of degree $n$, and if the $(p+1)$ th letter of this cycle, counting from the next to the last letter of the first cycle of $s_{2}$, does not appear in $s_{1}^{\prime}$ we adjoin to $s_{1}^{\prime}$ a transposition composed of this letter and the next to the last letter in the first cycle of $s_{2}$. If the said $(p+1)$ th letter appears in $s_{1}^{\prime}$, the preceding letter cannot have this property, and hence we begin our cycle with the third letter from the end of the first cycle and adjoin to $s_{1}^{\prime}$ the transposition composed of this letter and the $p$ th letter in the said cycle of order $n$. In both cases we obtain a value of $s_{1}^{\prime}$ such that $s_{2} s_{1}^{\prime}$ involves a cycle of order $p$, and that we can add another transposition to $s_{1}^{\prime}$, in case we desire to change its sign, without affecting this cycle of order $p$. When $s_{2}$ involves only one cycle, the $k$ letters which do not appear in $s_{2}$ may be connected with the first $k$ letters of $s_{2}$ and we may begin our cycle of order $n$ with the last letter of $s_{2}$. The $(p+1)$ th letter of this cycle cannot now appear in $s_{1}^{\prime}$ and hence we may adjoin to $s_{1}^{\prime}$ the transposition composed of this letter and the last letter of $s_{2}$ in order to obtain a cycle of order $p$ in the product of $s_{2} s_{1}$; and an additional transposition can be added to this $s_{1}$ without affecting this cycle. When $l$ is a power of 2 which is divisible by 8 , similar considerations obviously apply, and hence it remains only to consider the case where $l=4$.

While an infinite number of exceptions presented themselves when $l>3$ was assumed to be an odd prime number, one for each such prime, there is only one exception when $l=4$, since every alternating group which involves substitutions of order 4 can be generated by two operations of orders 2 and 4 respectively, and every symmetric group, except the symmetric group of degree 6 , can be generated by two such operators whenever its order is divisible by 4 . To prove this theorem it may be assumed that $s_{2}$ is positive and that its degree is $n-k, k<2$, and that $s_{2}$ involves at most three transpositions. When $k=1$ the letter which is not found in $s_{2}$ is connected by $s_{1}^{\prime}$ with the last letter of $s_{2}$, while $s_{1}^{\prime}$ connects the other letters of $s_{2}$ as in the preceding cases so that $s_{2} s_{1}^{\prime}$ is again a single cycle of order $n$. When $n>19, s_{2}$ involves at least four cycles of order 4 , and when $k=0, s_{1}^{\prime}$ is positive when the transposition is adjoined to give a cycle of order $p$ in $s_{2} s_{1}^{\prime}$. Hence when 
$p=n-3$ it is not necessary to adjoin to $s_{1}^{\prime}$ an additional transposition to obtain generators of the alternating group. Generators of the symmetric group in this case may be obtained by replacing a cycle of order 4 in $s_{2}$ by two transpositions. When $p<n-3$ it is clearly possible to assume that $s_{2}$ is always positive and to adjoin a transposition to $s_{1}^{\prime}$ without affecting the cycle of order $p$. Hence two substitutions of orders 2 and 4 respectively can always be found so that they generate either the alternating or the symmetric group of degree $n$ as may be desired whenever $n>19$.

When $7<n<20$ the value of $p$ can be so selected as to make the determination of two possible generators $s_{1}, s_{2}$ very simple. The groups of degrees 6 and 7 are so well known that it seems unnecessary to give here two substitutions of orders 2 and 4 respectively which generate the alternating groups of these degrees or the symmetric group of degree 7 as may be desired. On the other hand, it may be of some interest to give an outline of a proof that the symmetric group of degree 6 cannot be thus generated. If it could be generated by two such substitutions we may assume that $s_{2}$ would be one of the following two substitutions, $a b c d, a b c d \cdot e f$. Since the separate groups generated by these substitutions are transformed into themselves by a group of order 16 on these letters we need to use for $s_{1}$ only one of each set of conjugates under this group. When $s_{2}$ is the former of the two given substitutions $s_{1}$ may therefore be assumed to be one of the following four substitutions:

$$
c e \cdot d f, \quad b e \cdot d f, \quad a b \cdot c e \cdot d f, \quad a c \cdot b e \cdot d f .
$$

In the first case the commutator of $s_{2}$ and $s_{1}$ would be adcef. This commutator and $s_{2}{ }^{2}$ generate the simple group of order 60 since their product is of order 3. As this group is invariant under $s_{1}$ and $s_{2}$, these two substitutions generate the triply transitive group of order 120 . The second and fourth substitutions to be used for $s_{1}$ evidently generate an imprimitive group with $s_{2}$, while the third and $s_{2}$ again generate the triply transitive group of order 120 , since $s_{1} s_{2}$ in this case is acedf and the square of this into $s_{2}{ }^{2}$ is again of order 3. Hence these two substitutions generate the simple group of order 60 which is invariant under $s_{1}$ and $s_{2}$.

When $s_{2}=a b c d$. ef it may be assumed that $s_{1}$ is one of the following three substitutions:

$$
d e, \quad a b \cdot c f \cdot d e, \quad a c \cdot b f \cdot d e .
$$

In the first case it follows from a general theorem noted above the $s_{1}$ and $s_{2}$ generate a group of order 72 . In the other two cases it is obvious that they must also generate an imprimitive group. Hence it has been proved that the symmetric group of degree 6 can not be generated by two of its substitutions of orders 2 and 4 respectively. That is, 
Every symmetric group whose order is divisible by 4 except the symmetric group of degree 6 can be generated by two operators of orders 2 and 4 respectively, and every alternating group which involves operators of order 4 can be generated by two such operators.

UNIVERSTTY OF ImInNOIS, Urbana, III. 